\title{
Human Mesenchymal Stem Cells (MSC) Indirectly Preserve Liver of Irradiation Damage
}

\author{
Moubarak Mouiseddine ${ }^{1}$, Sabine François ${ }^{1}$, Alexandra Semont ${ }^{1}$, Amandine Sache ${ }^{1}$ Christelle \\ Demarquay $^{1}$, Anne Athias ${ }^{2}$, Patrick Gourmelon ${ }^{1}$, Norbert-Claude Gorin ${ }^{3}$, Maâmar Souidi ${ }^{1}$ and \\ Alain Chapel ${ }^{1, *}$ \\ ${ }^{1}$ Institut of Radioprotection and Nuclear Safety (IRSN), BP $n^{\circ}$ 17, F-92262 Fontenay aux Roses cedex \\ ${ }^{2}$ Institut National de la santé et de la Recherche Médicale (INSERM), Unité 498, hôpital du bocage 21079 Dijon cedex, \\ ${ }^{3}$ Institut National de la santé et de la Recherche Médicale (INSERM), Unité 392, Université Pierre et Marie Curie, \\ Paris. France
}

\begin{abstract}
The present work was initiated in an effort to evaluate the potential therapeutic contribution of the infusion of MSC for the correction of liver injuries.

We subjected NOD-SCID mice to a 10.5 Gy abdominal irradiation and we tested the biological and histological markers of liver injury in the absence and after infusion of expanded human MSC.

Irradiation alone induced a significant elevation of transaminases (ALT and AST). Apoptosis in the endothelial layer of vessels was observed. When MSC were infused in mice, a significant decrease of transaminases was measured, and a total disappearance of apoptotic cells. MSC were not found in liver. To explain the protection of liver without MSC engraftment, we hypothesize an indirect action of MSC on the liver via the intestinal tract. Pelvic or total body irradiation induces intestinal absorption defects leading to an alteration of the enterohepatic recirculation of bile acids. This alteration induces an increase in Deoxy Cholic Acid (DCA) which is hepatoxic. In the present study, we confirm these results. DCA concentration increased approximately 2-fold after irradiation but stayed to the baseline level after MSC injection. We propose from our observations that, following irradiation, MSC infusion indirectly corrected liver dysfunction by preventing gut damage. This explanation would be consistent with the absence of MSC engraftment in liver.
\end{abstract}

These results evidenced that MSC treatment of a target organ may have an effect on distant tissues. This observation comes in support to the interest for the use of MSC for cellular therapy in multiple pathologies proposed in the recent years.

Keywords: Mesenchymal stem cells, liver, human.

\section{INTRODUCTION}

Mesenchymal stem cells (MSC) are described as multipotent progenitor cells that differentiate into osteocytes, chondrocytes, adipocytes, and stromal cells [1-3]. MSC have been successfully used in therapeutics $[4,5]$ to correct ostegenesis imperfecta [6], to improve hematopoiesis [7-9] and to prevent Graft Versus Host Disease post hemopoietic stem cell transplantation [10-12].

In addition to these therapeutical domains, MSC have recently received attention for their potential as regenerative medicine following radiation injuries. The ileum of irradiated pigs shows many alterations such as blunting and/or villi loss. Radiation-induced alterations of intestinal absorption have been previously described [13, 14].

*Address correspondence to this author at the IRSN, DRPH/SRBE, BP17, F-92262 Fontenay aux roses, France; Tel: 33(1)58359546;

Fax: 33(1)58358467; E-mail: alain.chapel@irsn.fr
In the rat model, it has been shown that radiation induces acute alterations of the enterohepatic recirculation which concomitantly with radiation-induced intestinal malabsorption, leads to alterations of hepatic synthesis and secretion [15]. In NOD-SCID mice, intestinal radiation injury is characterized by impaired epithelial renewal, leading to mucosal disruption and functional abnormalities [16]. We have previously described the capacity of MSC to restore intestinal integrity after radiation induced damage [17]. We also have shown that MSC when infused to mice that received either extended or localized irradiation, migrate to almost all tissues where they engraft transiently usually at very low levels of detection [18]. Observations in the liver of human transplant recipients have shown a long-term implantation of a very small number of donor bone marrow cells which might be of mesenchymal origin [19]. MSC improve liver function and modulate hepatocellular death. These effects may be mediated by hepatocytes replacement and/or secretion of growth factors [20-22]. 
In the present work we found that human MSC, when injected after abdominal irradiation in NOD-SCID mice, were able to indirectly preserve the liver of radiation damage. We studied the mechanism of this protection. Following our previous findings on intestinal protection, we found that the MSC regenerated the small intestine epithelium, which in turn restored the entero-hepatic recirculation pathway initially damaged by irradiation. The consequence was a distant hepatic protection without engraftment of MSC in liver.

\section{MATERIAL AND METHODS}

\section{Culture of Human MSC}

Bone marrow (BM) cells were obtained from iliac crest aspirates of healthy volunteers after informed consent and were used in accordance with the procedures approved by the human experimentation and ethic committees of the Hôpital St Antoine. As previously described. Fifty milliliters of BM were taken from different donors in the presence of heparin (Sanofi-synthélabo, France). Lowdensity mononuclear cells were separated on the Ficoll Hypaque density gradient (d 1.077). Mononuclear cells (MNC) were plated at a density of $1.3310^{6}$ cells $/ \mathrm{cm} 2$ corresponding to a concentration of $10^{7}$ cells $/ 10 \mathrm{ml}$ of McCoy's $5 \mathrm{~A}$ medium supplemented with $12.5 \%$ foetal calf serum, $12.5 \%$ horse serum, $1 \%$ sodium bicarbonate, $1 \%$ sodium pyruvate, $0.4 \%$ MEM non essential amino acids, $0.8 \%$ MEM essential amino acids, 1\% MEM vitamin solution, 1\% L-glutamine (200 mM), 1\% penicillinstreptomycin solution (all from Invitrogen, Groningen, The Netherlands), $10^{-6} \mathrm{M}$ hydrocortisone (Stem Cell Technologies ${ }^{\circledR}$ ), 2 ng/ml human basic recombinant fibroblast growth factor (R\&D System, Abington, UK) in $\mathrm{T}-75 \mathrm{~cm}^{2}$ tissue culture flasks and incubated at $37^{\circ} \mathrm{C}$ in humidified, $5 \% \mathrm{CO}_{2}$ atmosphere. After three days, non-adherent cells were washed with PBS and fresh medium (without hydrocortisone) was added. Samples of human MSC from different donors were collected at the second passage for transplantation.

\section{FACS Analysis}

Before injection, the MSC phenotype was checked. Stainings were performed with phycoerythrin (PE)conjugated monoclonal antibody against CD105 (SH2), CD73 (SH3), and CD45 (Becton, Dickinson and Company, Franklin Lakes, NJ, http://www.bd.com) for 30 minutes at $4^{\circ} \mathrm{C}$ followed by two washes in PBS containing $0.5 \%$ BSA. Cells were re-suspended in $200 \mu \mathrm{l}$ of PBS, $0.5 \%$ BSA, and analyzed at 10,000 events per test by FACScalibur BD (Pharmingen, San Diego,CA, USA). Mouse immunoglobulin G1 (IgG1) was used as an isotopic control (Beckman Coulter, Fullerton, CA, USA).

\section{Irradiation}

Mice were irradiated locally at the abdominal area using an ICO4000 device (Cobalt 60 source). The window of irradiation is two centimeter of width on tree centimeter of length. The dose rate is 2 Gy per minute. After anaesthesia mice were maintained on target during the time of irradiation. Each mouse was separately irradiated at the dose of 10.5 Gy.

\section{NOD/SCID Mice Model}

All experiments and procedures were performed in accordance with the French Ministry of Agriculture regulations for animal experimentation (Act $n^{\circ} 87-847$ October 19th 1987, modified May 2001) and were approved by the animal care committee of the Institut de Radioprotection et de Sûreté Nucléaire (IRSN). NOD-LtSzscid/scid (NODSCID) mice, from breeding pairs originally purchased from Jackson Laboratory (Bar Harbor, ME, USA) were bred in our pathogen-free unit and maintained in sterile micro isolator cages. A total of 25 twelve-week-old mice divided into 4 groups were used for this study. Two groups received IV a dose of $5.10^{6}$ human MSC. Group 1 was a non-irradiated control group and did not receive human MSC. Group 2 was not irradiated and received MSC infusion. Group 3 was irradiated at a sub lethal dose of 10.5 Grays (Gy) and did not receive MSC infusion. Group 4 was irradiated at a sub lethal dose of 10.5 Gy and received MSC infusion 5 hours later.

\section{PCR}

The animals were sacrificed five-days after irradiation. Peripheral blood, liver and kidneys, were collected and the quantitative implantation of human MSC in mouse tissue was defined by real time PCR experiments as previously described [21]. Briefly human beta globine gene was amplified from DNA in order to detect human cell engraftment; mouse RAPSYN gene was used as control of amplification.

\section{Histology}

Liver and kidney alterations were studied by histological analysis on day five. Formalin-fixed, paraffin-embedded liver and kidney from NOD/SCID mice were cut at $5 \mu \mathrm{m}$ on a rotary microtome (Leica Microsystems AG, Wetzlar Germany) and mounted on polysine slides. Sections were deparaffinized in xylene and rehydrated with ethanol and PBS. Sections were stained with haematoxylin, eosin and safran (HES).

\section{Tunnel Assay}

Apoptotic cells were determined using the in situ cell Death Detection kit from Roche Diagnostics (Mannheim, Germany) following the manufacturer's instructions. The apoptotic cells (brown staining) were counted under a microscope. The apoptotic index was defined by the percentage of brown (dark) cells among the total number of cells in each sample. Five fields with 100 cells per field were randomly counted for each sample. We counted a minimum of three samples, thus making a total of fifteen single analyses.

\section{Plasma Analysis}

Animals were anesthetized by Rompan-Imalgene solution and killed by intracardiac puncture with a 1-ml insulin syringe to collect blood. The abdomen was opened and the liver and kidney were rapidly excised, weighed and apportioned for preparing cellular fractions or storage at $-80^{\circ} \mathrm{C}$ for future use. Blood was collected in $1.5 \mathrm{ml}$ tubes with heparin syringe to prevent serum formation by in vitro coagulation. Samples were centrifuged at $8000 \mathrm{rpm}$ at $4{ }^{\circ} \mathrm{C}$ for 5 min to collect the plasma. We used an automated Konelab 20 apparatus (Thermo Electron Corporation, Courtaboeuf, 
France) system to measure plasma alanine amino-transferase (ALT), aspartate amino-transferase (AST), (biological chemistry reagents, Bayer Diagnostics) in the control and different groups of mice.

\section{Quantification of Liver Bilic Acids}

Liver samples were previously homogenised in $150 \mathrm{mM}$ $\mathrm{NaCl}$. The procedure of sample extraction is adapted from Keller and al. Nor-cholic acid was used as the internal standard. Analysis of bilic acids (BA) was performed by Gas Chromatography-Mass Spectrometry (GC-MS).

\section{Statistical Analysis}

All values were expressed as the mean and SEM (Standard Error of the Mean). To compare results between groups, we used a $\mathrm{T}$ test or a one-way ANOVA followed by Tukey test with Sigmastat software (Systat Software Incorporation). Significance for all analyses was set at $* * * \mathrm{P}<0.001$ or $* * \mathrm{P}<0.05$.

\section{RESULTS}

\section{MSC Characterisation}

Phenotypic analysis showed that the MSC used in these experiments following expansion, were strongly positive for the specific surface antigens $\mathrm{SH} 2$ and $\mathrm{SH} 3$, respectively $37.3 \% \pm 4.0$ and $72.9 \% \pm 3.7$. Almost no contamination $(0.2 \% \pm 0.1 \mathrm{CD} 45+$ cells $)$ by haematopoietic cells was detected (Table 1). MSC had their specific fibroblast-like appearance. Before use, we checked that each batch of MSC retained its specific ability to undergo osteogenic, chondrogenic and adipogenic differentiation. Our results suggest that the MSC used in these experiments for transplant were expanded without significant loss in their differentiation capacities.

\section{MSC were not Found in Liver}

Human MSC were tracked in liver and gut at five-days after injection using real time PCR. MSC engrafted is small intestine $(0.2 \% \pm 0.03)$ but not in liver.

\section{MSC have no Hepatotoxic Potential}

There was no significant variation in ALT $(108 \pm 18.9$ $\mathrm{U} / \mathrm{I})$ and $\operatorname{AST}(20.3 \pm 0.8 \mathrm{U} / \mathrm{I})$ levels when we compared control mice with mice receiving $\mathrm{MSC}(\mathrm{ALT}=113 \pm 43.5$ $\mathrm{U} / \mathrm{I}, \mathrm{AST}=17.4 \pm 1.7 \mathrm{U} / \mathrm{I})$. (Fig. 1a and 1b).

\section{Irradiation has A Hepatotoxic Effect}

Abdominal irradiation resulted in a significant elevation of the ALT $(353.9 \pm 37.5 \mathrm{U} / \mathrm{I}$ vs. $108.6 \pm 18.9 \mathrm{U} / \mathrm{I})$ and AST $(40.3 \pm 4.9 \mathrm{U} / \mathrm{I}$ vs. $20.3 \pm 0.8 \mathrm{U} / \mathrm{I})$ levels after five-days $(\mathrm{p}<0.001)$ (Fig. 2a and 2b). Therefore, hepatic function was altered five-days after abdominal irradiation. By TUNEL marking on the liver before and after irradiation, we observed that irradiation induced apoptosis of the endothelial cells and the cells lining the bile ducts which are polarised hepatocytes. Irradiation induced damage to the liver (Fig. 3a. and 3b).

Table 1.

\begin{tabular}{|c|c|c|c|c|c|}
\hline No & BM ml & MSC (10 ${ }^{6}$ Cell)s & \% CD73 & \% CD105 CD45 \\
\hline \hline 1 & 25.0 & 37.5 & 65.9 & 38.1 & 43.1 \\
\hline 2 & 20.0 & 79.0 & 72.5 & 48.6 & 0.3 \\
\hline 3 & 8.5 & 20.5 & 71.6 & 27.7 & 0.3 \\
\hline 4 & 30.0 & 19.4 & 67.7 & 37.9 & 0.1 \\
\hline 5 & 32.0 & 58.5 & 72.9 & 37.3 & 0.2 \\
\hline Mean & 23.1 & 43.1 & 3.7 & 4.0 & 0.1 \\
\hline
\end{tabular}

Sample analysis and phenotypic characterization of cultured-expanded human MSC: Phenotypic analysis of MSC was performed at the second passage, before transplant. Frequency of positive cells for specific markers of hMSC: SH2 (CD105) and SH3 (CD73) and haematopoietic cell markers (CD45).

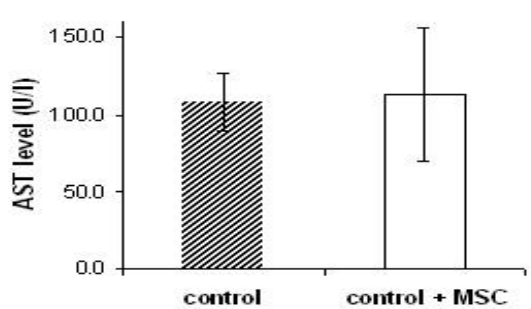

(a)

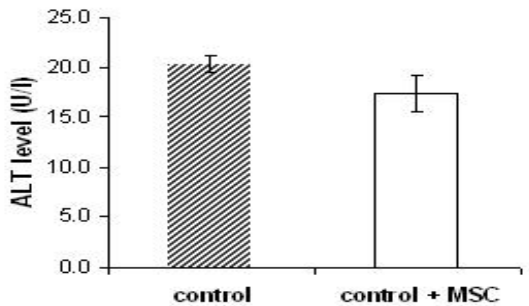

(b)

Fig. (1). Absence of toxicity of human MSC infused to NOD/SCID mice. Hatched histograms correspond to control animals non irradiated receiving no MSC ( $n=7)$. White histograms correspond to non irradiated animals receiving MSC ( $=4)$. There was no difference between the two groups for plasma levels of transaminases AST (a) and ALT (b). 


\section{MSC Protect the Liver After Irradiation}

MSC infusion prevented AST $(198.8 \pm 43.1 \mathrm{U} / \mathrm{I})$ and ALT $(14.9 \pm 4.7 \mathrm{U} / \mathrm{I})$ from increasing five-days after irradiation ( $\mathrm{p}<0.05$ and $\mathrm{p}<0.01$ respectively). (Fig. $\mathbf{2 a}$ and $\mathbf{2 b}$ ).

\section{MSC Injection Limits Liver Apoptosis (Figure 3c)}

Five-days after irradiation, apoptosis was investigated in the liver. In the untreated group irradiation induced apoptosis in the endothelial layer of vessels was observed $(29 \% \pm 7$ of apoptotic cells). However, when mice were transplanted with MSC the apoptotic areas disappeared $(3 \% \pm 2$ apoptotic cells, $\mathrm{P}<0.001$ compared to untreated group, Table 2 ).

\section{MSC Protect the Entero-Hepatic Recirculation Pathway}

Intestinal malabsorption is associated with an alteration of the enterohepatic recirculation of bile acids. In Fig. (4) we only present the variation of the most hydrophobic acid, DCA, in so far as the others did not vary significantly. After abdominal irradiation, bile acid concentrations were disturbed. The results show that DCA concentration increased approximately 2-fold. In contrast, after MSC injection the concentration of DCA equalled the control level. MSC therefore can prevent a five-days increase in deoxycholic acid concentration. MSC can correct the radioinduced disturbances which cause the variation of DCA. Since bile acids are regulated by the bowel, MSC can restore intestinal absorption of DCA.

\section{DISCUSSION}

The present work was initiated in an effort to evaluate the potential therapeutic contribution of the infusion of MSC for the correction of liver injuries induced by an irradiation exposure.

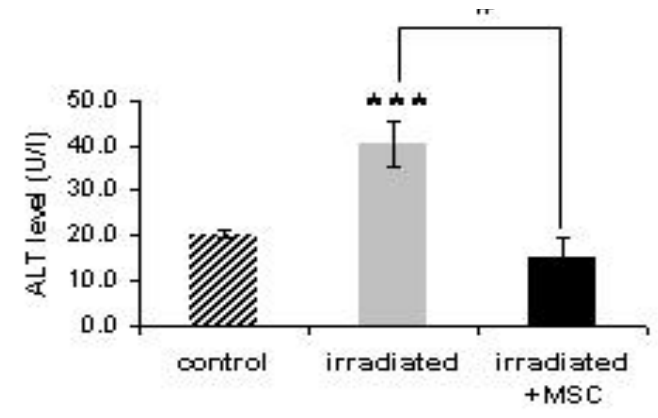

Fig. (2). Effect of irradiation and MSC infusion on liver. Hatched histograms correspond to non irradiated control animals receiving no MSC $(n=7)$. Grey histograms correspond to irradiated animals (dose: 10.5 Gy) with no MSC infusion ( $\mathrm{n}=5$ ). Black histograms correspond to animals receiving MSC after irradiation $(\mathrm{n}=8)$. Plasma dosages were done on day 5 post irradiation. (a) Transaminases AST: a significant increase in the AST plasma concentration was observed which was multiplied by 3 -fold $(\mathrm{p}<0.001)$. MSC infusion resulted in a significant reduction of the increase $(\mathrm{p}<0.05)$. (b) Transaminases ALT: a significant increase in the ALT plasma concentration was observed which was multiplied by 2 -fold $(\mathrm{p}<0.01)$. MSC infusion resulted in a significant reduction of the increase $(\mathrm{p}<0.05)$.
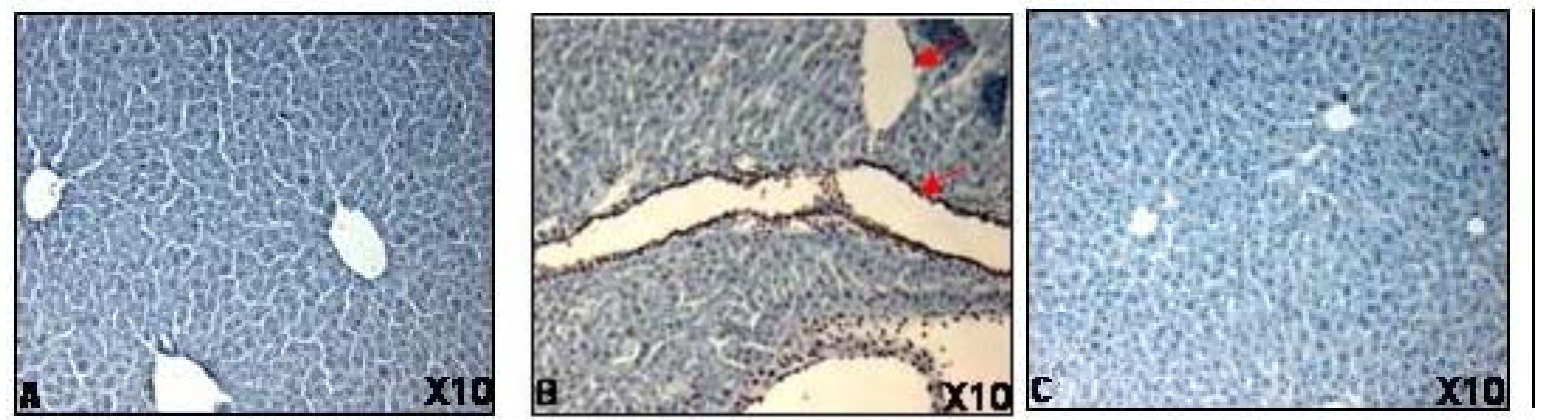

Fig. (3). Evaluation of apoptosis in the liver five-days after irradiation followed or not by MSC infusion. TUNEL staining: apoptotic and necrotic cells appear brown-coloured (red arrows). (A) Control, the necrotic and apoptotic cells seen after irradiation alone (B) were not seen in animals receiving MSC $(\mathbf{C})$ post irradiation (Magnification x10).

Table 2.

\begin{tabular}{|c|c|c|c|c|c|}
\hline \% of Apoptotic Cells & \multicolumn{3}{|c|}{ Irradiated } & \multicolumn{2}{c|}{ Irradiated + MSC } \\
\hline \hline 5 days after irradiation & Mean & SEM & $\mathrm{n}$ & Mean & SEM \\
\hline Liver & 29 & 7 & 5 & 3 & 2 \\
\hline
\end{tabular}

Percentage of apoptotic cells five-days after irradiation in liver. In the untreated group (Irradiated), irradiation induced apoptosis. In mice transplanted with MSC (irradiated + MSC) the apoptotic cells disappeared ( $\mathrm{P}<0.001$ compared to untreated group). 


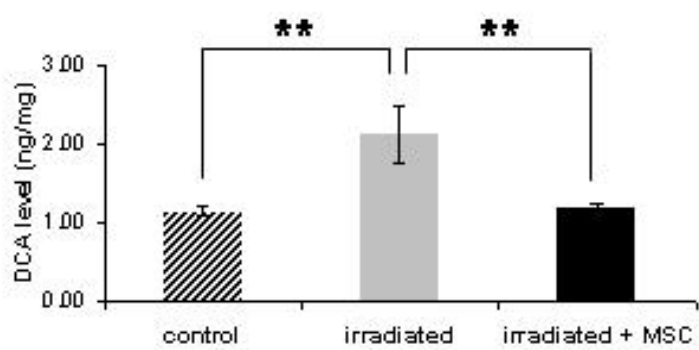

Fig. (4). Deoxycholic acid (DCA) variation five-days after irradiation followed or not by MSC infusion. Hatched histograms correspond to non irradiated control animals $(n=7)$, grey histograms to irradiated animals, black histograms to animals receiving MSC after irradiation $(\mathrm{n}=11)$. DCA concentration in liver increased significantly $(\mathrm{p}<0.01)$, by a factor of 2 , five-days after irradiation. After infusion of MSC, DCA concentration did not increase. The significant result for irradiated versus irradiated and treated is $\mathrm{p}<0.01$.

We subjected NOD-SCID mice to a 10.5 Gy abdominal irradiation and we tested the biological and histological markers of liver injury in the absence and after infusion of expanded human MSC. Irradiation alone induced a significant elevation of the ALT and AST. Necrosed endothelial cells and the cells lining the bile ducts were observed. Infusion of MSC into animals five hours after irradiation induced a significant decrease of transaminases, and an almost total disappearance of apoptotic cells. An effect of MSC with a decrease of apoptosis has however been described in a different situation, namely acute renal failure (ARF) [23].

To explain the restoration of liver damage as evaluated by the correction of transaminase levels and the decreasing number of apoptotic cells in the liver, as well as the absence of detectable implantation of MSC in the liver at five-days after infusion, we hypothesize an indirect action on the liver via the intestinal tract. Total body irradiation and abdominal irradiation induce structural and functional gut damage [23], $[16,24]$ with a decrease of villi height [17] and malabsorption [15]. Intestinal malabsorption in turn induces an alteration of the enterohepatic recirculation of bile acids [15], with an increase in Deoxy Cholic Acid (DCA) which is described as one of the most cytotoxic of them [25]. Indeed, in the present study, DCA concentration increased approximately 2-fold after irradiation but went back to the baseline level after MSC. We propose that, following irradiation, MSC infusion indirectly corrected liver dysfunction by preventing gut damage. One mechanism for this indirect effect is the alteration of the entero-hepatic recirculation pathway. Another mechanism that should be considered is the role of cytokines and growth factors produced by the MSC that are homing to other organs. Recent publications by VanPoll et al. (Hepatology 2008) showed in a model of fulminant hepatic failure (FHF) suggest that the infusion of conditioned medium from MSC protects from apoptosis in the liver and stimulate hepatocyte proliferation [20]. The indirect effect of MSC on the liver reported in the present study could correspond to a similar mechanism. This explanation would be consistent with the absence of detection of MSC in the liver. We believe our observation brings an additional piece of evidence in favour of the use of MSC in patients submitted to pelvic or total body irradiation.

\section{AUTHOR DISCLOSURE STATEMENT}

No competing financial interests exist.

\section{REFERENCES}

[1] Friedenstein AJ, Gorskaja JF, Kulagin NN. Fibroblast precursors in normal and irradiated mouse hematopoietic organs. Exp Hematol 1976; 4: 267-74.

[2] Prockop DJ. Marrow stromal cells as stem cells for nonhematopoietic tissues. Science 1997; 276: 71-4.

[3] Pittenger MF, Mackay AM, Beck SC, et al. Multilineage potential of adult human mesenchymal stem cells. Science 1999; 284: 143-7.

[4] Lazarus HM. Bone marrow transplantation in low-grade nonHodgkin's lymphoma. Leuk Lymphoma 1995; 17: 199-210.

[5] Koc ON, Peters C, Aubourg P, et al. Bone marrow-derived mesenchymal stem cells remain host-derived despite successful hematopoietic engraftment after allogeneic transplantation in patients with lysosomal and peroxisomal storage diseases. Exp Hematol 1999; 27 : 1675-81.

[6] Horwitz EM, Gordon PL, Koo WK, et al. Isolated allogeneic bone marrow-derived mesenchymal cells engraft and stimulate growth in children with osteogenesis imperfecta: implications for cell therapy of bone. Proc Natl Acad Sci USA 2002; 99: 8932-7.

[7] Koc ON, Gerson SL, Cooper BW, et al. Rapid hematopoietic recovery after coinfusion of autologous-blood stem cells and cultureexpanded marrow mesenchymal stem cells in advanced breast cancer patients receiving high-dose chemotherapy. J Clin Oncol 2000; 18: 307-16.

[8] Fouillard L, Bensidhoum M, Bories D, et al. Engraftment of allogeneic mesenchymal stem cells in the bone marrow of a patient with severe idiopathic aplastic anemia improves stroma. Leukemia 2003; 17: 474-6.

[9] Fouillard L, Chapel A, Bories D, et al. Infusion of allogeneicrelated HLA mismatched mesenchymal stem cells for the treatment of incomplete engraftment following autologous haematopoietic stem cell transplantation. Leukemia 2007; 21: 568-70.

[10] Lazarus HM, Loberiza FR, Zhang MJ, et al. Autotransplants for Hodgkin's disease in first relapse or second remission: a report from the autologous blood and marrow transplant registry (ABMTR). Bone Marrow Transplant 2001; 27: 387-96.

[11] Le Blanc K, Rasmusson I, Sundberg B, et al. Treatment of severe acute graft-versus-host disease with third party haploidentical mesenchymal stem cells. Lancet 2004; 363: 1439-41.

[12] Ringden O, Uzunel M, Rasmusson I, et al. Mesenchymal stem cells for treatment of therapy-resistant graft-versus-host disease. Transplantation 2006; 81: 1390-7.

[13] Thomson AB, Cheeseman CI, Walker K. Effect of external abdominal irradiation on the dimensions and characteristics of the barriers to passive transport in the rat intestine. Lipids 1984; 19: 405-18.

[14] Yeoh E, Horowitz M, Russo A, et al. Effect of pelvic irradiation on gastrointestinal function: a prospective longitudinal study. Am J Med 1994; 95: 397-406.

[15] Scanff P, Grison S, Marais T, et al. Dose dependence effects of ionizing radiation on bile acid metabolism in the rat. Int $\mathrm{J}$ Radiat Biol 2002; 78: 41-7.

[16] Francois S, Bensidhoum M, Mouiseddine M, et al. Local irradiation not only induces homing of human mesenchymal stem cells at exposed sites but promotes their widespread engraftment to multiple organs: a study of their quantitative distribution after irradiation damage. Stem Cells 2006; 24: 1020-9.

[17] Semont A, Francois S, Mouiseddine M, et al. Mesenchymal stem cells increase self-renewal of small intestinal epithelium and accelerate structural recovery after radiation injury. Adv Exp Med Biol 2006; 585: 19-30.

[18] Bensidhoum M, Chapel A, Francois S, et al. Homing of in vitro expanded Stro-1- or Stro-1+ human mesenchymal stem cells into the NOD/SCID mouse and their role in supporting human CD34 cell engraftment. Blood 2004; 103: 3313-9. 
[19] Korbling M, Katz RL, Khanna A, et al. Hepatocytes and epithelial cells of donor origin in recipients of peripheral-blood stem cells. N Engl J Med 2002; 346: 738-46.

[20] Poll DV, Parekkadan B, Cho CH, et al. Stem cell-derived molecules directly modulate hepathocellular death and regeneration in vitro and in vivo. Hepathology 2008; 47: 1634-43.

[21] Banas A, Teratani T, Yamamoto Y, et al. IFATS collection: in vivo therapeutic potential of uman adipose tissue mesenchymal stem cells after transplantation into mice with liver injury. Stem cells 2008; 26: 2705-12.

[22] Togel F, Hu Z, Weiss K, et al. Administered mesenchymal stem cells protect against ischemic acute renal failure through differen- tiation-independent mechanisms. Am J Physiol Renal Physiol 2005; 289: F31-42.

[23] Quastler H. The nature of intestinal radiation death. Radiat Res 1956; 4: 303-20.

[24] Francois A, Milliat F, Vozenin-Brotons MC, et al. 'In-field' and 'out-of-field' functional impairment during subacute and chronic phases of experimental radiation enteropathy in the rat. Int J Radiat Biol 2003; 79: 437-50.

[25] Hofmann AF. The continuing importance of bile acids in liver and intestinal disease. Arch Intern Med 1999; 159: 2647-58.

(C) Mouiseddine et al.; Licensee Bentham Open.

This is an open access article licensed under the terms of the Creative Commons Attribution Non-Commercial License (http://creativecommons.org/licenses/by-nc/3.0/) which permits unrestricted, non-commercial use, distribution and reproduction in any medium, provided the work is properly cited. 